\title{
CONECTIVIDADE FUNCIONAL E A IMPORTÂNCIA DA INTERAÇÃO ORGANISMO-PAISAGEM*
}

\author{
German Forero-Medina ${ }^{1}$ \& Marcus Vinícius Vieira ${ }^{1}$ \\ ${ }^{1}$ Laboratório de Vertebrados, Departamento de Ecologia, Instituto de Biologia - CCS, Universidade Federal do Rio de Janeiro, CEP:21941-590. Rio de \\ Janeiro, Brasil. \\ E-mails: forecroc@yahoo.com, mvvieira@biologia.ufrj.br.
}

\section{RESUMO}

Devido à crescente taxa de modificações antrópicas na paisagem, que resultam na fragmentação e perda de habitat, é importante estudar os efeitos que mudanças na configuração espacial têm nos processos ecológicos. A conectividade, o inverso da fragmentação, determina o grau no qual uma paisagem facilita ou restringe o movimento dos organismos entre fragmentos. Ela influencia a sobrevivência das populações e a dinâmica populacional em paisagens fragmentadas. Com o desenvolvimento da Ecologia da Paisagem apareceram diversos índices para medir o isolamento ou conectividade da paisagem. Alguns dos índices foram criticados por serem redundantes, sensíveis à escala, e por medir aspectos estritamente estruturais da conectividade. Diversos autores têm chamado a atenção sobre a importância de considerar a interação entre o organismo e a estrutura da paisagem para avaliar a conectividade. Existe uma diferença entre conectividade estrutural, que só considera aspectos físicos da paisagem, e conectividade funcional, que leva em conta a interação organismopaisagem. A conectividade funcional é uma variável dependente da capacidade de dispersão do organismo. As prioridades de pesquisa devem ser enfocadas em estudar aspectos comportamentais próprios de cada espécie e fazer uma ligação entre estas características específicas e a conectividade da paisagem. A teoria dos grafos é uma ferramenta útil, que permite usar informação biológica de cada espécie e tomar decisões para melhorar a conectividade funcional da paisagem.

Palavras-chave: Fragmentação de habitats, dispersão, capacidade perceptual, teoria dos grafos.

\begin{abstract}
FUNCTIONAL CONECTIVITY AND THE IMPORTANCE OF THE LANDSCAPE-ORGANISM INTERACTION. The effects that changes on landscape spatial patterns have on ecological processes are of great importance due to the increasing rate of human modifications on the landscape, such as habitat fragmentation and habitat loss. Landscape connectivity, the opposite of fragmentation, determines the degree to which a landscape facilitates or restricts the movement of organisms between habitat patches. It influences population survivorship and dynamics on fragmented landscapes. With the development of Landscape Ecology, different indices for estimating landscape connectivity appeared. Some indices were criticized because of their redundancy, sensibility to scale, and because they measured only physical aspects of the landscape. Several authors have called attention on the importance of considering organismlandscape interactions when studying connectivity. There is a difference between structural connectivity, which considers only physical aspects of the landscape, and functional connectivity, which considers species-landscape interactions. Functional connectivity is a variable dependent on the vagility of the organism. Research priorities should focus on studying species-specific behavioral aspects, and making a link between these traits and landscape connectivity. Graph theory appears as a useful tool for studying functional connectivity. It allows for the use of species specific traits, and it helps on decision making for increasing landscape connectivity.
\end{abstract}

Keywords: Habitat fragmentation, dispersal, perceptual range, graph theory 


\section{INTRODUÇÃO}

A fragmentação de habitats é uma das mais citadas causas da extinção das espécies e perda da diversidade biológica (D’Eon et al. 2002), sendo um dos tópicos de conservação mais estudados e discutidos nos últimos anos. Pode ser definida de forma geral como o processo pelo qual uma área continua de habitat é reduzida em tamanho, e dividida em dois ou mais fragmentos separados por um entorno ou 'matriz' de habitats diferentes do original (Wilcove et al. 1986). Segundo esta definição, o processo de fragmentação tem quatro efeitos sobre o padrão do uso do habitat: 1) redução na extensão de habitat original, 2) aumento no número de fragmentos de habitat, 3) diminuição no tamanho dos fragmentos, e 4) aumento no isolamento dos fragmentos (Fahrig 2003).

Devido aos efeitos da perda de habitat original serem diferentes e independentes dos efeitos da fragmentação per se, foi sugerido que os dois processos fossem denominados e estudados de maneira independente, sendo a fragmentação restringida às mudanças na configuração do habitat resultantes da divisão do mesmo, independentes da perda de área (Fahrig 2003). Porém, na maioria dos estudos sobre fragmentação, não é feita uma distinção entre os dois processos e os efeitos da perda de hábitat não são controlados na avaliação dos efeitos da fragmentação per se. Isto dificulta determinar em muitos casos se os mencionados efeitos negativos da fragmentação são devidos realmente à fragmentação, à perda de habitat ou à interação dos dois processos.

Ao diminuir uma área continua em fragmentos de tamanho menor, dentro de uma 'matriz' de habitat não favorável, o número de espécies em cada fragmento deverá acompanhar a diminuição na área, reduzindose até ajustar-se ao novo tamanho de cada fragmento. Este fenômeno é conhecido como relaxation times (Diamond 1972, Newmark 1987). Além disso, populações isoladas em fragmentos muito pequenos terão uma probabilidade de persistência reduzida, devido à diminuição no tamanho populacional e aos efeitos dos processos estocásticos (Gilpin \& Soulé 1986). A diminuição do tamanho dos fragmentos de habitat original, por outro lado, aumenta a relação perímetroárea, incrementando as conseqüências negativas dos efeitos de borda. Entre estes efeitos negativos encontram-se, além das mudanças microclimáticas (Love- joy et al. 1986), o aumento da atividade dos predadores nas bordas (Chalfoun et al. 2002), maior probabilidade dos indivíduos de passar do fragmento para a 'matriz', aumentando assim a mortalidade devido às condições potencialmente desfavoráveis do ambiente da 'matriz' (Fahrig 2002), e maior probabilidade de invasão de espécies exóticas (Simberloff 1993).

A fragmentação e perda de habitat têm outros efeitos negativos sobre a biodiversidade. Além de afetar a riqueza de espécies (Gurd et al. 2001, Steffan-Dewenter et al. 2002) podem afetar também a abundância e distribuição das populações (Hanski et al. 1996). A perda de habitat pode afetar a taxa de crescimento populacional (Bascompte et al. 2002), diminuir o comprimento e a diversidade da cadeia trófica (Komonen et al. 2000, Dobson et al. 2006), alterar interações entre espécies (Taylor \& Merriam 1996), afetar espécies que dependem de recursos efêmeros (Gibbs \& Stanton 2001), e diminuir o sucesso na dispersão (Bélisle et al. 2001). A fragmentação também pode afetar negativamente o sucesso reprodutivo e a taxa de predação (Kurki et al. 2000).

\section{CONECTIVIDADE DA PAISAGEM}

Durante o processo da fragmentação, o potencial de dispersão e colonização de uma espécie pode ser limitado pela criação de barreiras (Primack 1998). Esta pode ser uma conseqüência grave, porque a habilidade dos animais de mover-se entre fragmentos é um dos fatores mais importantes na dinâmica populacional em paisagens fragmentadas (Zollner \& Lima 1999b). Neste sentido, a conectividade, o inverso da fragmentação, é considerada um elemento vital da paisagem já que é crítica para a sobrevivência da população e para a dinâmica populacional (Fahrig \& Paloheimo 1988). Pode ser definida como o grau no qual uma paisagem facilita ou restringe o movimento entre fragmentos (Taylor et al. 1993).

A diminuição da conectividade, ao limitar a dispersão dos organismos, pode ter consequiências negativas nas populações já que reduz o fluxo genético entre elas. Isto pode levar a endocruzamento e perda de diversidade genética, como tem sido encontrado já em ambientes fragmentados (Gibbs 2001). A conectividade da paisagem é importante então para o fluxo genético entre populações (Coulon et al. 2004). Processos como os movimentos entre fragmentos e 
fluxos ecológicos em geral tem sido considerados como prioridades de investigação na Ecologia da Paisagem (Wu \& Hobbs 2002).

\section{ECOLOGIA DA PAISAGEM E A MEDIÇÃO DA CONECTIVIDADE}

Devido às modificações crescentes do homem na paisagem (Wilcove 1986) é importante entender como funcionam diferentes processos biológicos em paisagensheterogêneas, eainda comoestes sãoafetados por mudanças de origem antrópica na paisagem. A Ecologia da Paisagem é uma disciplina que estuda o desenvolvimento e dinâmica da heterogeneidade espacial, as interações espaciais, temporais e trocas através de ecossistemas heterogêneos, as influências da heterogeneidade espacial nos processos bióticos e abióticos, e o manejo da heterogeneidade espacial (Barret \& Peles 1999). Uma das prioridades da Ecologia da Paisagem é identificar e entender as relações entre padrões espaciais e processos ecológicos (Wu \& Hobbs 2002, Rutledge 2003), o que permite entender melhor o efeito das alterações antrópicas na paisagem.

Devido à reconhecida importância de estudar padrões espaciais e processos ecológicos, foram desenvolvidos nos últimos anos muitos índices para medir a estrutura da paisagem (Tischendorf 2001). Estes índices, ou métricas da paisagem, podem agrupar-se em dois grupos, índices não espaciais ou de composição e espaciais ou de disposição (Rutledge 2003, Metzeger 2004). Os primeiros descrevem o número de unidades e a proporção da área ocupada por elas, os últimos descrevem os atributos das manchas e proporcionam informação relevante à medição da fragmentação (Rutledge 2003).

Uma das características da paisagem comumente estudadas é precisamente a conectividade. Esta variável é medida usando um conjunto de índices espaciais, que medem o grau de isolamento ou conectividade das manchas. Os índices de isolamento podem ser divididos em duas classes (Metzeger 2004): os que medem o isolamento de um único fragmento usando medidas de distância entre fragmentos, e os que medem o isolamento médio na paisagem, usando medidas de proximidade entre pixels. Já os índices de conectividade medem a densidade de estruturas de conexão (corredores, stepping stones), a proximidade ou percolação das áreas de hábitat, ou a permeabilidade da 'matriz' (Metzeger 2004).

Porém, o uso de índices da paisagem tem sido reavaliado recentemente, pois segundo alguns autores, em geral os índices não servem como indicadores dos efeitos da fragmentação e não necessariamente estão correlacionados com processos ecológicos (Rutledge 2003, Tischendorf 2001, Taylor et al. no prelo). Muitos índices estão correlacionados entre si, proporcionando assim informação redundante sobre a estrutura da paisagem, enquanto alguns outros são sensíveis à escala em que são medidos (Rutledge 2003, Tischendorf 2001). Os índices são uma ferramenta importante e útil para descrever e comparar padrões espaciais (Rutledge 2003), e alguns índices estão correlacionados com processos ecológicos como a dispersão, mas só em algumas situações (Tischendorf 2001). Assim, estas correlações são muitas vezes inconsistentes e ambíguas para diferentes estruturas da paisagem ou comportamentos de dispersão, variando de relações altamente positivas a altamente negativas, dependendo do caso (Tischendorf 2001).

$\mathrm{O}$ estudo da conectividade então se limita geralmente ao aspecto estrutural, e as medidas de conectividade usadas com mais freqüência enfocam principalmente a área dos fragmentos e as distâncias entre eles (Taylor et al. no prelo). Porém, a conectividade não pode ser definida baseando-se simplesmente nas distâncias entre manchas já que a conectividade é o resultado da interação entre um processo comportamental (movimento) e a estrutura física da paisagem. É uma propriedade dinâmica estudada na escala da paisagem para um determinado organismo, e não uma propriedade agregada de um conjunto de manchas de habitat (Taylor et al. no prelo).

\section{CONECTIVIDADE ESTRUTURAL VS. CO- NECTIVIDADE FUNCIONAL}

Podem ser distinguidos dois tipos de conectividade, estrutural e funcional. A primeira descreve relações físicas entre as manchas, como distâncias entre elas e corredores. É baseada completamente na estrutura da paisagem, ignorando as respostas dos organismos (Taylor et al. no prelo). A conectividade funcional, por outro lado, considera as respostas comportamentais aos elementos da paisagem junto com a estrutura espacial (Goodwin 2003). A conectividade pode ser 
vista então como uma variável independente, que tem efeitos sobre os processos ecológicos e as populações, ou como uma variável dependente da interação entre estrutura e comportamento (Goodwin 2003).

A mobilidade de um organismo é um dos determinantes mais importantes da conectividade funcional da paisagem (D’Eon et al. 2003), e esta última tem que ser considerada na escala da interação entre a paisagem e o organismo em questão. Já que os diferentes organismos respondem à heterogeneidade ambiental em diferentes escalas e em diferentes formas (Johnson et al. 1992), uma paisagem não é inerentemente fragmentada ou conectada. A conectividade só pode ser determinada no contexto da capacidade do organismo de se mover entre fragmentos (D’Eon et al. 2003). Geralmente não é possível extrapolar a conectividade funcional da conectividade estrutural (Taylor et al. no prelo) (Figura 1).

Esta visão concorda com a perspectiva do 'modelo de paisagem continuo' (continua-umwelt model) (Manning et al. 2004), que difere do tradicional 'modelo de fragmentação'. O modelo de estudo da paisagem chamado de 'modelo de fragmentação' teve sua origem na teoria de biogeografia de ilhas e considera as manchas de habitat como ilhas dentro de uma 'matriz' não habitável (Fischer \& Lindenmayer 2006). Este modelo assome que existe um claro contraste entre as manchas e o resto da paisagem, e que vários organismos percebem as manchas definidas pelo homem como manchas de habitat favorável (Fischer \& Lindenmayer 2006). Porém em algumas paisagens não existe uma diferença clara entre manchas e 'matriz', e algumas espécies usam a matriz, chegando inclusive a ser favorecidas pela criação de áreas abertas. O modelo contínuo, pelo contrário, reconhece gradientes espaciais, estados graduais de alteração da paisagem: habitat intacto, variegado, fragmentado ou relictual. Este modelo também reconhece que os diferentes organismos percebem e respondem ao ambiente de formas diferentes (Manning et al. 2004). Assim, o grau de uso de um determinado habitat por uma espécie varia geralmente de forma continua e não de forma categórica como habitat ou não habitat. Estas diferenças no gradiente de uso de habitats pelas espécies determinam a distribuição delas nos remanescentes de habitat original, e nos habitats de entorno.

A conectividade funcional de uma paisagem pode referir-se então à ligação funcional entre fragmentos de habitat, bem seja porque esses estão conectados através de uma continuidade estrutural, porque os organismos usam o habitat de 'matriz', ou porque as habilidades de dispersão dos organismos lhes permitem se deslocar entre fragmentos discretos, percebendo-os como funcionalmente conectados a

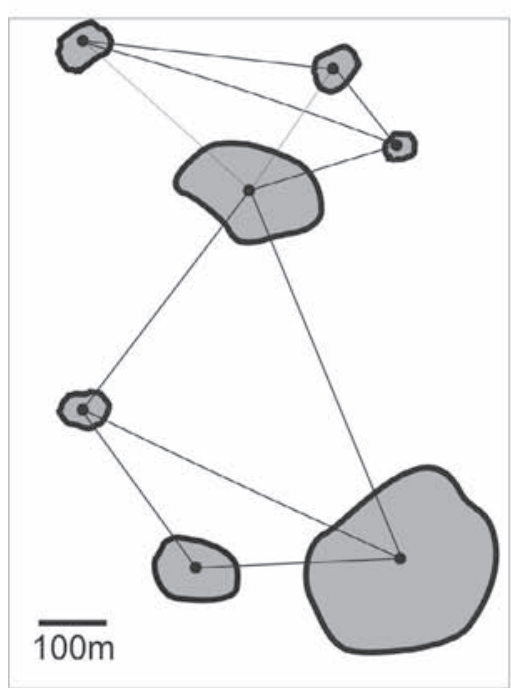

b

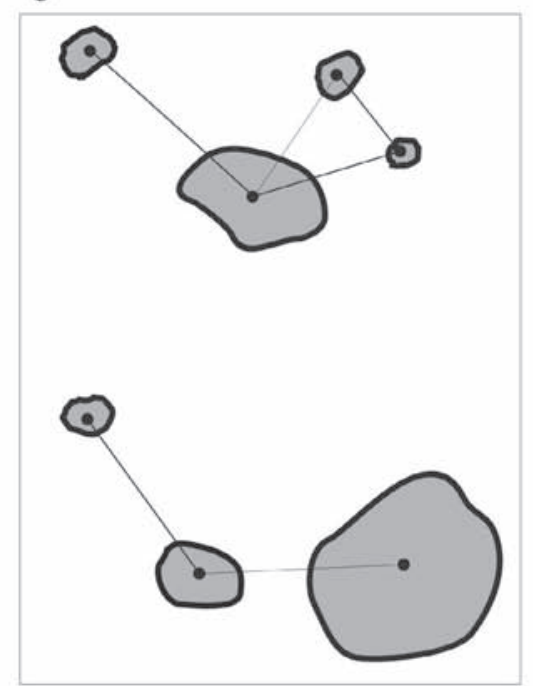

c

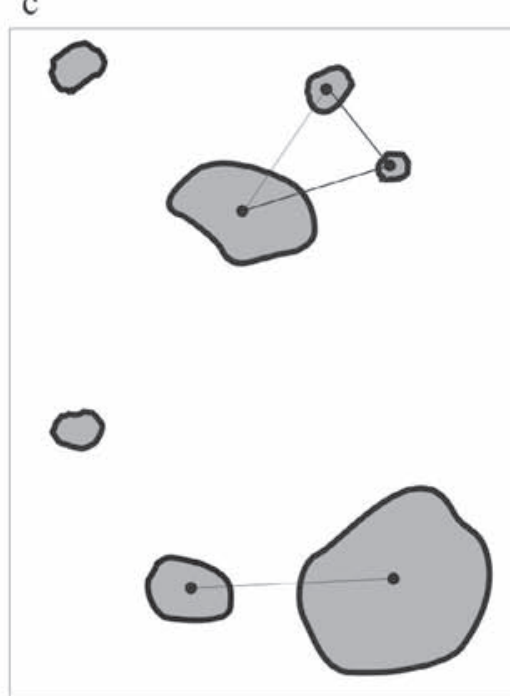

Figura 1. Conectividade funcional de uma paisagem para três espécies com capacidades diferentes de atravessar o habitat de ‘matriz’: a) 400m b) 200m e c) $100 \mathrm{~m}$. As linhas entre manchas indicam que a espécie pode atravessar de um fragmento a outro. A conectividade estrutural desta paisagem, medida a través de índices de isolamento ou conectividade apresentaria um valor único para qualquer espécie. Porém, a conectividade funcional é diferente para cada uma. Para a espécie da Figura 1a, a paisagem apresenta a maior conectividade funcional, estando completamente conectada. Para a espécie da Figura $1 \mathrm{~b}$ a paisagem tem duas áreas de habitat conectadas, mas isoladas entre si. Para a espécie da Figura 1c, a paisagem está fragmentada em quatro pequenas áreas de habitat isoladas entre elas. 
(With \& King 1999). Fatores como a mortalidade durante a dispersão, e aspectos da história de vida como tamanho do corpo e capacidade de deslocamento, podem influenciar a distribuição dos organismos em paisagens fragmentadas (Taylor et al. 1993, Zollner \& Lima 1999a). Por outro lado, a capacidade de movimentação entre remanescentes de habitat original, como característica comportamental, estará determinada pelas restrições fisiológicas e morfológicas de cada espécie, assim como pelas capacidades sensoriais.

Lima \& Zollner (1996) têm chamado a atenção sobre a necessidade de estabelecer uma ligação entre a ecologia comportamental e a ecologia da paisagem. Diversos trabalhos com modelagem na escala da paisagem estão baseados em fenômenos comportamentais pouco conhecidos. Estes fenômenos incluem movimentos e seleção de habitat, áreas nas quais a ecologia comportamental tem trabalhado bastante. Porém, o problema de aplicar a Ecologia Comportamental à Ecologia da Paisagem, segundo Lima \& Zollner (1996), consiste em que os pesquisadores destas duas áreas trabalham em escalas espaciais diferentes. Estes autores propõem que uma maior ligação entre estas duas áreas pode contribuir à compreensão, na escala da paisagem, da informação disponível para um animal em quanto se desloca em seu ambiente, e como esta informação é usada na seleção de uma mancha ou um habitat.

\section{PERSPECTIVAS}

Recentemente têm aumentado o número de estudos sobre conectividade funcional que consideram características dos organismos, como uso e capacidade de atravessar a 'matriz' (Tischendorf \& Fahrig 2000, Bélisle et al. 2001, Tischendorf 2001, D’Eon et al. 2002, Goodwin \& Fahrig 2002). Estes estudos, em grande parte baseados em simulações ou experimentos com invertebrados, têm contribuído muito ao entendimento dos efeitos dos padrões espaciais nos movimentos na paisagem. Por exemplo, Goodwin \& Fahrig (2002) mostraram, usando uma combinação de modelagem e experimentos com besouros, que a conectividade diminui significativamente com o aumento da distância entre manchas, e que a influencia dos elementos da 'matriz' na conectividade é pequena comparada com a influencia dos elementos de habitat. Bélisle et al. (2001) encontraram que os movimentos de três espécies de aves neotropicais, Dendroica caerulescens, Seiurus aurocapillus e Poecile atricapillus, eram influenciados pela composição e configuração da paisagem, ressaltando a importância da interação entre o comportamento e a paisagem.

As simulações são uma ferramenta útil para determinar o efeito da estrutura da paisagem nos diferentes processos como dispersão e colonização de manchas. Não obstante, as simulações requerem, como mencionado anteriormente, informações sobre características dos organismos. O conhecimento de tais características é muito limitado, principalmente para vertebrados, e especialmente para os trópicos. Um melhor conhecimento sobre estas variáveis permitiria entender melhor os efeitos da fragmentação, e ajudaria na tomada de decisões orientadas a otimizar a conectividade funcional. Isto seria de grande importância considerando que existem biomas altamente fragmentados, como a Mata Atlântica brasileira, um dos 34 hotspots de biodiversidade reconhecidos mundialmente (Myers et al. 2000, Mittermeier et al. 2004), que atualmente tem menos de $8 \%$ da sua extensão original devido à fragmentação e perda de habitat (Pinto \& Brito 2005). As estratégias de conservação de áreas tão fragmentadas como a Mata Atlântica dependem então de informações sobre como os processos ecológicos e evolutivos podem ser mantidos nos fragmentos pequenos e alterados que ainda persistem (Pardini et al. 2005).

Dada a estreita relação entre padrões espaciais na paisagem e processos ecológicos, e o pouco conhecimento que ainda existe sobre estas relações, as prioridades de investigação devem estar direcionadas aos processos, e ao efeito da estrutura da paisagem neles (Wu \& Hobbs 2002). Um dos processos que devem ser estudados é o movimento dos organismos entre manchas de habitat. Estes movimentos podem estar influenciados por fatores como uso da 'matriz', mortalidade durante a dispersão, comportamentos sociais, capacidade de locomoção (Taylor et al. 1993, Zollner \& Lima 1999a), e a capacidade de perceber um fragmento a distância (Zollner 2000).

Algumas perguntas contribuiriam para entender melhor os efeitos das mudanças na estrutura da paisagem sobre os movimentos dos organismos, e a modelar a conectividade funcional com mais precisão: $\mathrm{O}$ que faz com que um determinado organismo saia de uma mancha de habitat para outra usando a 'matriz'? 
Qual é a distância máxima que um organismo, usando uma determinada 'matriz', pode atravessar de um fragmento pata outro? Como determinado tipo de 'matriz' afeta a mortalidade e capacidade de locomoção de um organismo? O que faz com que um organismo permaneça ou não em uma mancha de habitat?

As respostas a algumas destas perguntas são mais difíceis de responder do que outras, tanto teórica quanto experimentalmente. Os pequenos mamíferos têm sido considerados como o grupo taxonômico ideal para responder perguntas em nível da paisagem, devido à relativa abundância de informação sobre sua biologia, à facilidade de captura e marcação, e ao tamanho relativamente pequeno das áreas que habitam (Barret \& Peles 1999). De fato, 8\% dos trabalhos publicados na revista Landscape Ecology têm enfocado pequenos mamíferos (Barret \& Peles 1999). Devido a isto, a discussão sobre as perspectivas para abordar perguntas na escala da paisagem estará baseada principalmente em pequenos mamíferos. Porém as idéias e métodos podem ser aplicados a diferentes grupos taxonômicos.

Para responder estas perguntas devem ser realizados estudos no hábitat original de cada espécie e na 'matriz'. Os diferentes estudos sobre movimentação e área de vida de pequenos mamíferos neotropicais, têm sido enfocados principalmente em movimentos dentro dos habitats das espécies, como floresta Atlântica ou restinga (Loretto \& Vieira 2005, Gentile \& Cerqueira 1995, Gentile et al. 1997, Cunha \& Vieira 2002, Cáceres 2003). Porém poucos trabalhos têm estudado os movimentos nas áreas de 'matriz', com influência antrópica, que estão ao redor das áreas de habitat favoráveis. Os trabalhos relacionados com movimentos na 'matriz' consistem principalmente de resultados de métodos de remoção ou captura-marcação-recaptura (Olifiers 2002, Pires et al. 2002) e em menor grau telemetria (Lira 2005).

Estes trabalhos mostraram que varias espécies são capturadas na 'matriz', e que os indivíduos podem se deslocar de um fragmento para outro. Não obstante, pouco é conhecido sobre o comportamento dos indivíduos no habitat de 'matriz', ou as variáveis bióticas ou ambientais que influenciam estes movimentos. As distâncias máximas de deslocamento entre fragmentos usando diferentes tipos de 'matriz' também não são conhecidas.

Um dos mecanismos comportamentais espécie- específicos que pode ser determinante no sucesso de dispersão é a capacidade de um organismo de perceber um habitat à distância (Zollner 2000). Lima \& Zollner (1996) definiram a capacidade perceptual (perceptual range) como a distância desde a qual um elemento da paisagem pode ser percebido ou detectado por um determinado animal. A capacidade perceptual de um animal determina a facilidade de localizar fragmentos de habitat, e pelo tanto o tempo de procura numa ‘matriz' não favorável (Zollner \& Lima 1999a). Um animal com uma estreita capacidade perceptual teráum risco de mortalidade relativamente alto, pois gastará mais tempo procurando um habitat favorável que um animal com uma distância perceptual ampla (Zollner \& Lima 1997). Portanto, a capacidade perceptual é um determinante importante da conectividade funcional da paisagem (Lima \& Zollner 1996) e da dinâmica da população em ambientes fragmentados (Zollner \& Lima 1999a). Da mesma maneira, a sensibilidade de uma espécie à fragmentação será uma função da sua capacidade perceptual (Zollner 2000).

Porém, há pouca informação empírica sobre a escala perceptual de vertebrados (Zollner \& Lima 1999a). As poucas espécies estudadas incluem a tartaruga de água doce Trachemys scripta, cuja capacidade perceptual foi determinada em 300m (Yeomans 1995), e os pequenos mamíferos Clethrionomys gapperi, que pode perceber habitat de floresta até aproximadamente $10 \mathrm{~m}$ (Gillis \& Nams 1998), Peromyscus leucopus, que em condições de luz tem uma escala perceptual de 90m (Zollner \& Lima 1999b), Taias striatus com aproximadamente $120 \mathrm{~m}$, Sciurus carolinensis com $300 \mathrm{~m}$, e Sciurus niger com 400m (Zollner 2000).

Os resultados de um trabalho recente com marsupiais didelfídeos da Mata Atlântica (ForeroMedina 2007) mostraram que indivíduos de varias espécies podem perceber fragmentos de habitat a diferentes distâncias, e orientar-se em direção a eles, quando se movimentam em uma 'matriz' de pasto. Uma das alternativas complementares aos trabalhos de captura-marcação-recaptura, é estudar a capacidade perceptual de algumas espécies, e usar estas distâncias de percepção para estudar a conectividade funcional da paisagem.

Esta abordagem permite também, através de experimentos em campo, estudar outras variáveis que influenciam a orientação dos organismos durante 
a procura de habitat, como o vento. O vento pode influenciar a orientação de algumas espécies, que se movem direcionadas a favor ou em contra dele, provavelmente porque usam elementos olfativos, ou usam o vento para manter uma trajetória reta na procura de habitat (Schooley \& Wiens 2003). Este comportamento é conhecido como anemotaxis (Schooley \& Branch 2005).

As pesquisas sobre movimentação em áreas fragmentadas, devem contemplar assim, estudos de marcação-recaptura, telemetria e estudos comportamentais. Os estudos de marcação-recaptura permitem conhecer a freqüência de movimentos entre fragmentos, os estudos de telemetria permitem conhecer as características dos movimentos e áreas de vida das diferentes espécies dentro e fora dos fragmentos. Os estudos comportamentais permitem entender melhor as causas de tais movimentos, e a informação disponível para os organismos durante a procura de habitat, como as variáveis que afetam a orientação e a capacidade de percepção de um habitat.

\section{TEORIA DO GRAFOS}

Uma abordagem relativamente recente em termos da conectividade da paisagem, que considera a informação sobre este tipo de distâncias de percepção ou deslocamento é a teoria dos grafos aplicada à ecologia (Urban \& Keitt 2001). Esta abordagem é relativamente simples, e pode servir como um guia importante nas decisões sobre a importância relativa de manchas individuais para a conectividade geral da paisagem.

A teoria dos grafos é conhecida e aplicada comumente em áreas como a geografia, tecnologia da informação e computação (Bunn et al. 2000). Suas aplicações nestas áreas estão relacionadas com a otimização do fluxo em redes ou circuitos, mas ela representa uma ferramenta importante nas análises de conectividade da paisagem (Urban \& Keitt 2001). A teoria dos grafos trabalha com um tipo de dados espaciais conhecido como grafo. Um grafo é um conjunto de nodos ou vértices conectados entre si, representado geralmente como um diagrama (Urban \& Keitt 2001). A Figura 1a é um exemplo de um grafo com sete nodos. Um grafo apresenta então uma paisagem como um conjunto de nodos conectados de alguma forma. Uma conexão entre dois nodos implica que existe algum tipo de fluxo ecológico entre eles, como dispersão de propágulos ou fluxo de material (Urban \& Keitt 2001). Um grafo está conectado se existe um caminho entre cada par de nodos, isto é se todo nodo é atingível desde qualquer outro nodo. Um grafo não conectado, pelo contrário, consiste de outros subgrafos. A Figura 1b mostra dois subgrafos, de quatro e três nodos.

O estudo da conectividade sob uma perspectiva dos grafos permite modelar a conectividade funcional da paisagem para uma determinada espécie avaliando o efeito de diferentes práticas ou variáveis através de operações com os grafos. Existem dois tipos de operações com grafos: i) as relacionadas com a conexão entre nodos, ii) e as relacionadas com os nodos (Urban \& Keitt 2001). Nas aplicações à ecologia da paisagem, as primeiras permitem avaliar o efeito de modificações nas conexões funcionais entre fragmentos, por exemplo, o uso de corredores. As segundas permitem estudar o efeito da perda ou ganho de fragmentos na conectividade funcional.

Estas aplicações são de grande utilidade. Por exemplo, é possível modelar a conectividade funcional de uma mesma paisagem para espécies com capacidades de dispersão diferentes. Isto pode ajudar a determinar a sensibilidade à fragmentação de cada espécie em uma paisagem determinada. Por outro lado, o segundo tipo de operações, permite determinar a importância, para a conectividade funcional da paisagem, de preservar ou criar um determinado fragmento. Dada uma determinada paisagem é possível, depois de modelar a conectividade funcional para uma espécie, retirar fragmentos de interesse e avaliar o efeito na conectividade da paisagem (Figura 2). A determinação da conectividade pode ser feita a través de métricas como o número de subgrafos, o número de nodos (ordem) do maior grafo, ou o diâmetro do grafo maior (Urban \& Keitt 2001).

Esta abordagem permite propor estratégias para a conservação das espécies, orientadas a melhorar sua dispersão entre os fragmentos, e pode ser útil na escolha de áreas prioritárias para conservação ou restauração em paisagens fragmentadas da Mata Atlântica. É uma alternativa relativamente simples, e tem a vantagem de conseguir fazer uma conexão entre o organismo e a paisagem. Assim, pode aproveitar informação das espécies obtidas em experimentos de campo. Os modelos iniciais, construídos usando somente distâncias, podem e devem ser aperfeiçoados 
a

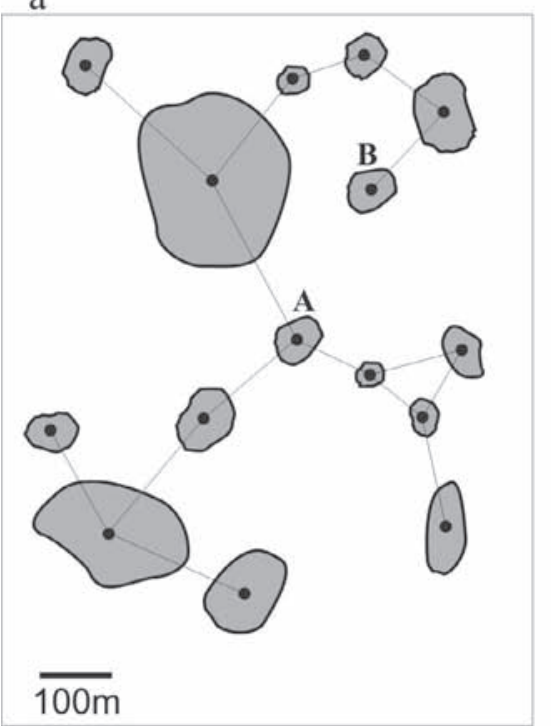

b

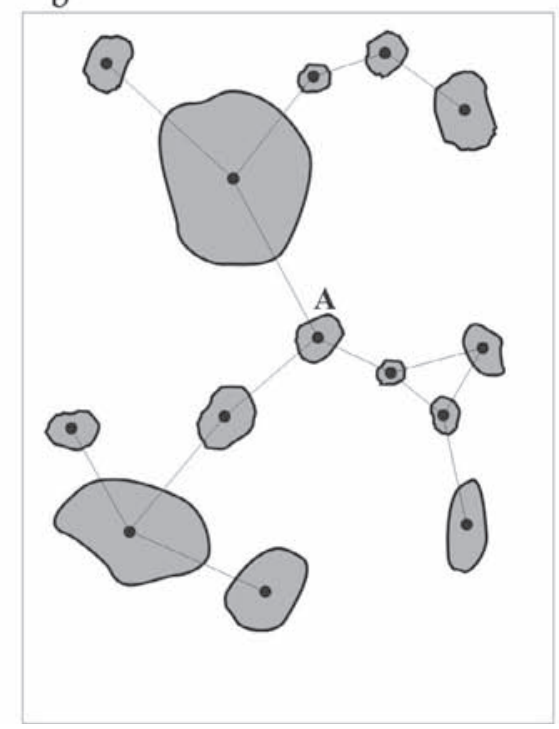

c

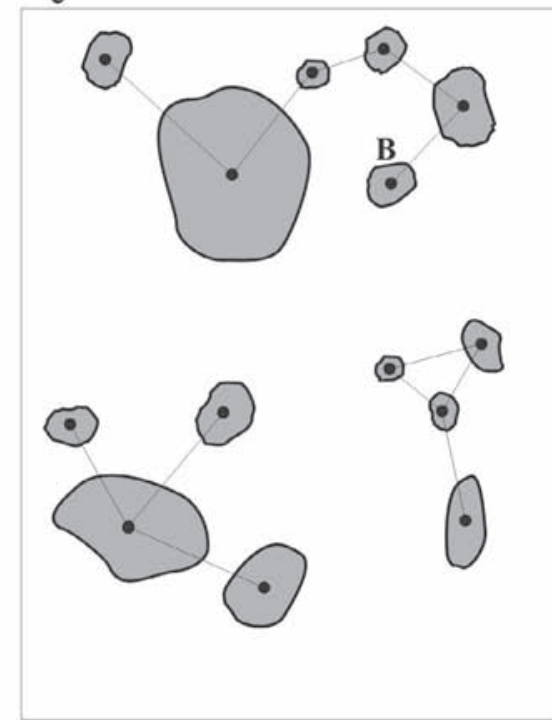

Figura 2. Conectividade funcional de uma paisagem para uma espécie com capacidade de dispersão entre fragmentos de 100m. a) Conectividade funcional da paisagem sem alterações b) Conectividade funcional se o fragmento B fosse removido, a paisagem se mantém com um único grafo c) Conectividade funcional se o fragmento A fosse removido, a conectividade funcional é menor, aparecendo agora três subgrafos. Ainda que apresentam a mesma área, o fragmento A é mais importante para a conectividade funcional da paisagem que o fragmento B.

posteriormente, incluindo mais informação como uso ou resistência da 'matriz', ou probabilidade de sair de um fragmento. Esta informação deve vir de estudos comportamentais orientados a proporcionar informação na escala da paisagem.

A conectividade funcional da paisagem é um dos aspectos mais relevantes e importantes na conservação, principalmente em áreas fragmentadas. Uma maior ligação entre a ecologia comportamental e a ecologia da paisagem, que estimule o estudo das interações organismo-paisagem, contribuirá ao desenvolvimento de propostas e estratégias orientadas a melhorar a conectividade. Só assim será possível garantir a sobrevivência das populações nas áreas de habitat cada vez menores que restam.

AGRADECIMENTOS: Ao Laboratório de Vertebrados, Programa de Pós-Graduação em Ecologia da UFRJ, CAPES e FAPERJ por incentivar e apoiar o trabalho. A Andréa Venturotti pelos comentários e ajuda com as figuras.

\section{REFERÊNCIAS}

BARRET, G.W. \& PELES, J.D. 1999. Landscape ecology of small mammals. Springer, New York.

BASCOMPTE, J.; POSSINGHAM, H. \& ROUGHGARDEN, J. 2002. Patchy populations in stochastic environments: Critical number of patches for persistence. The American Naturalist, 159: 128-137.
BÉLISLE, M.; DESROCHERS, A. \& FORTIN, M.-J. 2001. Influence of forest cover on the movements of forest birds: a homing experiment. Ecology, 87: 1893-1904.

BUNN, A.G.; URBAN, D.L. \& KEITT, T.H. 2000. Landscape connectivity: a conservation application of graph theory. Journal of Environmental Management, 59: 265-278.

CÁCERES, N.C. 2003. Use of the space by the opossum Didelphis aurita Wied-Newied (Mammalia, Marsupialia) in a mixed forest fragment of southern Brazil. Revista Brasileira de Zoologia, 20: 315-322.

CHALFOUN, A.D.; THOMPSON III, F.R. \& RATNASWAMY, M.J. 2002. Nest predators and fragmentation: a review and meta-analysis. Conservation Biology, 16: 306-318.

COULON, A.; COSSON, J.F.; ANGIBAULT, J.M.; CARGNELUTTI, B.; GALAN, M.; MORELLET, N.; PETIT, E.; AULAGNIER, S. \& HEWISON, J.M. 2004. Landscape connectivity influences gene flow in a roe deer population inhabiting a fragmented landscape: an individual-based approach. Molecular Ecology, 13: 2841-4850.

CUNHA, A.A. \& VIEIRA, M.V. 2002. Support diameter, incline, and vertical movements of four didelphid marsupials in the Atlantic forest of Brazil. Journal of Zoology, 258: 419-426.

D’EON, R.G.; GLENN, S.M.; PARFITT, I. \& FORTIN, M.J. 2002. Landscape connectivity as a function of scale and organism vagility in a real forested landscape. Conservation Ecology, 6: 1-10.

DIAMOND, J.M. 1972. Biogeographic kinetics: Estimation of relaxation times for avifaunas of SouthWest Pacific Islands. 
Proceedings of Natural Academie of Sciences (USA). 69: 3199-3203.

DOBSON, A.; LODGE, D.; ALDER, J.; CUMMING, G.S.; KEYMER, J.; MCGLADE, J.; MOONEY, H.; RUSAK, J.A.; SALA, O.; WOLTERS, V.; WALL, D.; WINFREE, R. \& XENOPOULOS, M. 2006. Habitat loss, trophic collapse, and the decline of ecosystem services. Ecology, 87: 1915-1924.

FAHRIG, L. 2002. Effect of habitat fragmentation on the extinction treshold: a synthesis. Ecological Applications, 12: 346-353.

FAHRIG, L. 2003. Effects of Habitat Fragmentation on Biodiversity. Annu. Rev. Ecol. Syst., 34: 487-515.

FAHRIG, L. \& PALOHEIMO, J. 1988. Determinants of local population size in patchy habitats. Theoretical Population Biology, 34: 194-213.

FISHER, J. \& LINDENMAYER, B. 2006. Beyond fragmentation: the continuum model for fauna research and conservation in human-modified landscapes. Oikos, 112(2): 473-480.

FORERO-MEDINA, G.A. 2007. Capacidade Perceptual de Pequenos Mamíferos da Mata Atlântica e Implicações para a Conectividade Funcional de uma Paisagem Fragmentada. Dissertação de Mestrado, PPGE-UFRJ, IB. Rio de Janeiro. 94p.

GENTILE, R. \& CERQUEIRA, R. 1995. Movement patterns of five species of small mammals in a Brazilian restinga. Journal of Tropical Ecology, 11: 671-677.

GENTILE, R.; D’ANDREA, P.S. \& CERQUEIRA, R. 1997. Home ranges of Philander frenata and Akodon cursor in a Brazilian Restinga (Coastal Shrubland). Mastozoologia Naotropical, 4: 105-112.

GIBBS, J.P. 2001. Demography versus habitat fragmentation as determinants of genetic variation in wild populations. Biological Conservation, 100: 15-20.

GIBBS, J.P. \& STANTON, E.J. 2001. Habitat fragmentation and arthropod community change: carrion beetles, phoretic mites, and flies. Ecological Applications, 11: 79-85.

GILLIS, E.A. \& NAMS, V.O. 1998. How red-backed voles find habitat patches. Canadian Journal of Zoology, 76: 791-794.

GILPIN, M.E. \& SOULÉ, M.E. 1986. Minimum viable populations: processes of species extinctions. Pp. 19-34 In: M.E Soulé, (ed.), Conservation Biology The Science of Scarcity and Diversity. Sinauer Associates Inc., Sunderland.

GOODWIN, B.J. 2003. Is landscape connectivity a dependent or independent variable? Landscape Ecology, 18: 687-699.

GOODWIN, B.J. \& FAHRIG, L. 2002. How does landscape structure influence landscape connectivity? Oikos, 99: 552570.

GURD, D.B.; NUDDS, T.D. \& RIVARD, D.H. 2001. Conservation of mammals in eastern North American wildlife reserves: how small is too small. Conservation Biology, 15: 1355-1363.

HANSKI, I.; MOILANEN, A. \& GYLLENBERG, M. 1996. Minimum viable metapopulation size. American Naturalist, 147: 527-41.

JOHNSON, A.R.; WIENS, J.A.; MILNE, B.T. \& CRIST, T.O. 1992. Animal movements and population dynamics in heterogeneous landscapes. Landscape Ecology, 7: 63-75.

KOMONEN, A.; PENTTILÄ, R.; LINDGREN, M. \& HANSKI, I. 2000. Forest fragmentation truncates a food chain based on an old-growth forest bracket fungus. Oikos, 90: 119-126.

KURKI, S.; NIKULA, A.; HELLE, P. \& LINDÉN, H. 2000. Landscape fragmentation and forest composition effects on grouse breeding success in boreal forests. Ecology, 81: 19851997.

LIMA, S.L. \& ZOLLNER, P.A. 1996. Towards a behavioral ecology of ecological landscapes. Trends in Ecology and Evolution, 11: 131-135.

LIRA, P. K. 2005. Padrões espaciais de marsupiais em uma paisagem fragmentada de Mata Atlânticano estado do Rio de Janeiro. Dissertação de Mestrado, Programa de PósGraduação em Ecologia. Universidade Federaldo Rio de Janeiro, Rio de Janeiro.

LORETTO, D. \& VIEIRA, M.V. 2005. The effects of reproductive and climatic seasons on movements in the black-eared opossum (Didelphis aurita Wied-Neuwied, 1826). Journal of Mammalogy, 86(2): 287-293.

LOVEJOY, T. E.; BIERREGAARD JR., R.O.; RYLANDS, A.B.; MALCOLM, J.R.; QUINTELA, C.E.; HARPER, L.H.; BROWN JR., K.S.; POWELL, A.H.; POWELL, G.V.N.; SCHUBART, H.O.R. \& HAYS, M.B. 1986. Edge and other effects of isolation on Amazon forest fragments. Pp. 257-285. In: M.E. Soulé, (ed), Conservation Biology The Science of Scarcity and Diversity. Sinauer Associates Inc., Sunderland.

MANNING, A.D.; LINDENMAYER, D.B. \& NIX, H.A. 2004. Continua and umwelt: novel perspectives on viewing landscapes. Oikos, 104(3):621-628.

METZEGER, J.-P. 2004. Estrutura da paisagem: o uso adequado de métricas. Pp. 423-453. In: L. Cullen Jr., R. Rudran \& C. Valladares-Padua, (eds.), Métodos de estudos em biologia da conservação \& manejo da vida silvestre. Universidade Federal do Paraná, Curitiba.

MITTERMEIER, R.A.; GIL, P.R.; HOFFMAN, M.; PILGRIM, J.; BROOKS, T.; MITTERMEIER, C.G.; LAMOUREX, J. \& FONSECA G.A.B. 2004. Hotspots revisited. Earth's biologically richest and most endangered terrestrial ecorregions. CEMEX, Mexico.

MYERS, N.; MITTERMEIER, R.A.; MITTERMEIER, C.G.; 
FONSECA, G.A.B. \& KENT, J. 2000. Biodiversity hotspots for conservation priorities. Nature, 403: 853-858.

NEWMARK, W.D. 1987. A land-bridge island perspective on mammalian extinctions in western North American parks. Nature, 325: 430-432.

OLIFIERS, N. 2002. Efeitos da fragmentação florestal e estrutura do hábitat sobre as comunidades de pequenos mamíferos da Bacia do Rio Macacu, RJ. Dissertação de Mestrado. PósGraduação em Ecologia, Conservação e Manejo de Fauna. Universidade Federal de Minas Gerais, Belo Horizonte.81p. PARDINI, R.; MARQUES DE SOUZA, S.; BRAGA-NETO, R. \& METZEGER, J.-P. 2005. The role of forest structure, fragment size and corridors in maintaining small mammal abundance and diversity in an Atlantic forest landscape. Biological Conservation, 124: 253-266.

PINTO, L.P. \& BRITO, M.C.W. 2005. Dinâmica da perda da biodiversidade na Mata Atlântica brasileira: uma introdução. Pp. 27-30. In: C. Galindo-Leal \& Câmara I.G., (eds.), Mata Atlântica Biodiversidade, Ameaças e Perspectivas. Fundação SOS Mata Atlântica, Conservação Internacional, Belo Horizonte.

PIRES, A. S.; LIRA, P.; FERNANDEZ, F. A. S.; SCHITTINI, G. M. \& OLIVEIRA, L. C. 2002. Frequency of movements of small mammals among Atlantic Coastal Forest fragments in Brazil. Biological Conservation, 108: 229-237.

PRIMACK, R.B. 1998. Essentials of conservation biology. (Second Edition). Sinauer Associates, Sunderland.

RUTLEDGE, D. 2003. Landscape indices as measures of the effects of fragmentation: can pattern reflect process. DOC Science Internal Series, 98: 1-28.

SCHOOLEY, R.L. \& BRANCH, L.C. 2005. Limited perceptual range and anemotaxis in marsh rice rats Oryzomys palustris. Acta Theriologica, 50: 59-66.

SCHOOLEY, R.L. \& WIENS, J. A. 2003. Finding habitat patches and directional connectivity. Oikos, 102: 559-570.

SIMBERLOFF, D. 1993. Effect of fragmentation on some Florida ecosystems, and how to redress them. Pp.179-187. In: D.A., Saunders; R. J. Hobbs \& P. Ehrlich, (eds.), Nature Conservation 3. Reconstruction of fragmented ecosystems. Surrey Beatry \& Sons.

STEFFAN-DEWENTER, I.; MÜNZENBERG, U.; BÜRGER, C.; THIES, C. \& TSCHARNTKE, T. 2002. Scale-dependent effects of landscape context on three pollinator guilds. Ecology, 83: 1421-1432.

TAYLOR, P.D.; FAHRIG, L.; HENEIN, K. \& MERRIAM, G. 1993. Connectivity is a vital element of landscape structure. Oikos, 68: 571-573.

TAYLOR, P.D. \& MERRIAM, G. 1996. Habitat fragmentation and parasitism of a forest damselfly. Landscape Ecology, 11: 181-189.

TISCHENDORF, L. 2001. Can landscape indices predict ecological processes consistently? Landscape Ecology, 16: 235254.

TISCHENDORF, L. \& FAHRIG, L. 2000. How should we measure landscape connectivity? Landscape Ecology, 15: 633-641.

URBAN, D.L. \& KEITT, T.H. 2001. Landscape connectivity: a graph-theoretic perspective. Ecology, 82(5): 1205-1218.

WILCOVE, D.S.; MCLELLAN, C.H. \& DOBSON, A.P. 1986. Habitat fragmentationin the temperate zone. Pp. 237-256. In: Soulé, M. E. (ed.), Conservation Biology The Science of Scarcity and Diversity. Sinauer Associates Inc., Sunderland.

WITH, K.A. \& KING, A.W. 1999. Dispersal success on fractal landscapes: a consequence of lacunarity tresholds. Landscape Ecology, 4: 73-82.

WU, J. \& HOBBS, R. 2002. Key issues and research priorities in landscape ecology: An idiosyncratic synthesis. Landscape Ecology, 17: 355-365.

YEOMANS, R.S. 1995. Water-finding in adult turtles: random search or oriented behaviour? Animal Behaviour, 49: 977-987.

ZOLLNER, P.A. 2000. Comparing the landscape level perceptual abilities of forest sciurids in fragmented agricultural landscapes. Landscape Ecology, 15: 523-533.

ZOLLNER, P.A. \& LIMA, S.L. 1997. Landscape-level perceptual abilities in white-footed mice: perceptual range and the detection of forested habitat. Oikos, 80: 51-60.

ZOLLNER, P.A. \& LIMA, S.L. 1999a. Illumination and the perception of remote habitat patches by white-footed mice. Animal Behaviour, 58: 489-500.

ZOLLNER, P.A. \& LIMA, S.L. 1999b. Search strategies for landscape-level interpatch movements. Ecology, 80: 10191030.

Submetido em 20/12/2006 Aceito em 21/06/2007 